\title{
Colorectal cancer after negative colonoscopy in fecal immuno- chemical test-positive participants from a colorectal cancer screening program
}

\section{(ㄷ)(1) $\odot(9$}

\author{
Authors \\ Liseth Rivero-Sánchez ${ }^{1}$, Jaume Grau², Josep María Augé3 ${ }^{3}$ Lorena Moreno4 ${ }^{4}$ Angels Pozo², Anna Serradesanferm², \\ Mireia Díaz ${ }^{4}$, Sabela Carballal' ${ }^{1}$, Ariadna Sánchez ${ }^{1}$, Leticia Moreira ${ }^{1}$, Francesc Balaguer ${ }^{1}$, Maria Pellisé ${ }^{1}$, Antoni \\ Castells ${ }^{1}$, on behalf of the PROCOLON group
}

Institutions

1 Gastroenterology Department, Hospital Clinic of Barcelona, Centro de Investigación Biomédica en Red de Enfermedades Hepáticas y Digestivas (CIBERehd), Universitat de Barcelona, Institut d'Investigacions Biomediques August Pi i Sunyer (IDIBAPS), Barcelona, Spain

2 Preventive Medicine and Hospital Epidemiology Department, Hospital Clínic, Barcelona, Spain

3 Biochemistry Department, Hospital Clinic of Barcelona, Barcelona, Spain

4 Institut d'Investigacions Biomediques August Pi i Sunyer (IDIBAPS), Fundació Clínic per la Recerca Biomèdica, Barcelona, Spain

submitted 27.2.2018

accepted after revision $\quad$ 14.5.2018

Bibliography

DOI https://doi.org/10.1055/a-0650-4296 |

Endoscopy International Open 2018; 06: E1140-E1148

(c) Georg Thieme Verlag KG Stuttgart · New York

ISSN 2364-3722

Corresponding author

Dr María Pellisé, Department of Gastroenterology, Hospital

Clínic, Villarroel 170, 08036 Barcelona, Catalonia, Spain

Fax: +34-93-2275589

mpellise@clinic.cat

\section{ABSTRACT}

Background and study aims Colorectal cancer (CRC) risk after a positive fecal immunochemical test (FIT) and negative colonoscopy is unknown. We aimed to ascertain the cumulative incidence of post-colonoscopy colorectal cancer (PCCRC) and the manifestation of other lesions that could explain the test positivity in individuals with a negative colonoscopy in a population screening program.

Patients and method Observational study in participants from the first round of a CRC screening program (20102012) with positive-FIT ( $\geq 20 \mu \mathrm{g} / \mathrm{g}$ of feces) and negative colonoscopy (without neoplasia). A 42- to 76-month follow-up was performed searching in the National Health Service database and by a brief structured telephonic interview.

Results Of 2659 FIT-positive individuals who underwent colonoscopy, 811 (30.5\%) had a negative colonoscopy. Three PCCRC $(0.4 \%)$ were detected within 11-28 months and accelerated carcinogenesis was ruled out. Among those with normal colonoscopy, 32 (5\%) relevant lesions were detected at follow-up. One-third of them (11/32) were significant neoplasias: a gastric cancer, a small-bowel lymphoma, six advanced colorectal adenomas, and the three PCCRC. The 21 remaining lesions were inflammatory, vascular disorders, or non-advanced colorectal adenomas.

Conclusions The vast majority (95\%) of individuals did not present any subsequent lesion that could explain the FIT positivity. The very low incidence $(0.4 \%)$ and characteristics of PCCRC observed in our cohort reinforce the concept that, although a positive FIT preselects high risk individuals, a high quality colonoscopy is the paramount factor in preventing PCCRC. Improving quality standards of colonoscopy are required to strengthen the current CRC screening strategies.

\section{Introduction}

Colorectal cancer (CRC) is currently the third most incident cancer worldwide and the second highest cause of cancer-related death [1]. Fecal occult blood test (FOBT)-based population screening programs are aimed at reducing the CRC mortality $[2,3]$ based on the selection of those asymptomatic individuals with a higher risk of having advanced adenomas $(\geq 10 \mathrm{~mm}$, villous component or high grade dysplasia) or cancer, to subse- 
quently undergo colonoscopy. Several population-based studies have demonstrated an important reduction in long-term risk of CRC and mortality after a colonoscopy [4, 5]. However, the colonoscopy is not faultless. CRC still occurs after a negative colonoscopy and before the recommended surveillance $[6,7]$, the so-called colonoscopy interval CRC [8].

Fecal immunochemical tests (FIT) are immunoassays specific for intact human hemoglobin [9], recommended as first choice over guaiac-FOBT given their higher specificity for human blood superiority and better sensitivity for the detection of advanced colorectal neoplasia [10]. In the scenario of organized FIT-based screening programs, around $20-30 \%$ of individuals have a positive test followed by a negative colonoscopy [11]. These individuals are considered at null risk for CRC for the following 10 years [12]. However, it is well known that about $20 \%$ of colorectal adenomas are missed at colonoscopy [13] and that the adenoma detection rate of the endoscopist is inversely related to the incidence of interval CRC [14]. The recommendation of a 10 -year interval without screening in those individuals with a false positive result may create a concern among endoscopists worried about the possibility of having missed a significant lesion. The incidence of CRC after a positive FIT and negative colonoscopy has not been reported until now.

In the context of an organized FIT-based CRC screening program, we aimed to assess the cumulative incidence of CRC in individuals with positive FIT followed by a negative colonoscopy. Secondly, we aimed to identify other lesions that could explain the test positivity in this cohort.

\section{Patients and methods}

This observational study was carried out within a FIT-based organized population CRC Screening Program, in which all individuals aged 50-69 were invited to participate. Personal history of CRC, adenoma, or inflammatory bowel disease, a family history of hereditary or familial CRC (defined as those individuals with two first-degree relatives with CRC or one diagnosed before the age of 60), severe coexisting illness, colonoscopy performed within the last 5 years, previous colectomy, or a contraindication for colonoscopy were considered exclusion criteria for screening. In the present study, we included all individuals living in the referral area of the Hospital Clinic of Barcelona who participated in the first round of the screening program (from January 2010 to December 2012) and had a positive FIT result followed by a complete negative colonoscopy defined as the absence of CRC, adenomas or serrated polyps (excluding hyperplastic polyps $\leq 5 \mathrm{~mm}$ in the sigmoid colon or rectum).

\section{Study setting and data collection}

The FIT-based screening program consisted of a single stool sample analysis using the automated semi-quantitative OCSensor (Eiken Chemical, Japan), without specific dietetic or medical treatment restrictions. Participants were always warned that if menstruation, hemorrhoid or fissure bleeding were present, the stool sample collection must be postponed until after 3 days without macroscopic bleeding. Positive FIT was defined as a cutoff of $\geq 20 \mu \mathrm{g}$ of hemoglobin/mg of feces.
Once a positive FIT-result was obtained, the colonoscopy was performed within 1 to 2 months.

For bowel preparation, all patients were encouraged to adhere to a low-fiber/fat diet 3 days before the colonoscopy and bowel cleansing was carried out with $4 \mathrm{~L}$ of polyethylene glycol and electrolyte lavage solution (Solución Evacuante Bohm, Laboratorios Bohm S.A., Fuenlabrada, Madrid, Spain) in splitdose [15]. For patients with previous inadequate preparation, sodium picosulfate magnesium oxide and citric acid (CitraFleet, Casen-Fleet, Zaragoza, Spain) were added to Bohm for intensive bowel cleansing.

All colonoscopies were performed in the Hospital Clinic of Barcelona, a tertiary academic center that follows high quality standards [16], by 12 experienced endoscopists each having performed more than 400 colonoscopies per year and with a known high adenoma detection rate (i.e. $29.8 \%$ in primary colonoscopy screening and $47.1 \%$ in FIT-based screening) [17, 18]. Procedures were performed under spontaneous breathing deep sedation (propofol and remifentanil infusion) administered by trained nurses supervised by anesthesiologists in 40minute time slots. Standard definition (CF-Q160L/CF-Q165L; EVIS EXERA II processor; Olympus, Tokyo, Japan) or high definition (CF-H180AL/CF-HQ190L; EVIS EXERA III processor; Olympus, Tokyo, Japan) white-light endoscopes were used. Bowel cleansing was considered adequate (excellent or good) if Boston score was $\geq 6$ points ( $\geq 2$ by colonic segment). Examination was considered completed if cecal intubation was reached and a minimum of 6 minutes of withdrawal time was normally advised.

Colonoscopies and their respective pathology reports were reviewed weekly by a committee composed of expert gastroenterologists, endoscopists, and nurses before follow-up recommendations were dictated. In cases of inadequate bowel preparation or incomplete procedure, colonoscopies were rescheduled as necessary until an optimal examination (i.e. complete with adequate colonic preparation) was achieved.

Participants' baseline data were prospectively recorded in the CRC screening program database. Demographics, comorbidities, chronic treatment, FIT levels, and index colonoscopy findings were obtained from both the CRC screening program database and hospital medical records. From each individual included, we investigated the hospital's medical records and Catalonia's National Health Service database in order to find any medical consultation due to gastrointestinal disorders after the index colonoscopy. The latest mentioned database registers only those patients who require hospitalization and/or complementary tests in public health centers other than Catalonia. When reliable information was lacking or absent (e.g. individuals attended private health care centers or those moving out of Catalonia), a brief structured telephonic interview was performed. This study was approved by the Ethic and Clinic Investigation Committee from Hospital Clinic of Barcelona. All individuals provided informed consent to participate in the CRC screening program and to collect their personal data on the program's database. All individuals interviewed by telephone provided a recorded informed consent. 


\section{Definitions and study outcomes}

Post-colonoscopy CRC (PCCRC) was defined as those tumors that invade the submucosa layer or beyond [8] detected after the (index) negative colonoscopy until the end of study followup. The incidence of PCCRC was expressed as cumulative incidence, defined as the proportion of PCCRC diagnosed during the observation time (cases/all individuals with negative colonoscopy*100) expressed as a percentage. Also the cumulative rate of PCCRC was calculated by dividing the cases of PCCRC by the sum of the observation time (average in years) of the individuals with negative colonoscopy ("at risk") expressed as cases/person-years. We also calculated the rate of PCCRC as the proportion of "new" cancers among the total number of cancers detected in the index colonoscopy cohort [8].

At baseline, negative colonoscopies were categorized into colonoscopy with potentially bleeding lesions (those with a high chance of producing occult or macroscopic bleeding, such as angiodysplasia, radiation proctitis, ulcers, etc.) and normal colonoscopy (no lesions or lesions of almost null probability of bleeding, such as non-complicated diverticulosis, left sidelocated small hyperplastic polyps, and hemorrhoids without stigmata of recent hemorrhage).

At follow-up, a relevant lesion was defined as any neoplastic or non-neoplastic lesion throughout the gastrointestinal tract that could reasonably produce a macroscopic or occult bleeding. Definitions were established by author consensus prior to data acquisition, i. e. erosive esophagitis Los Angeles's grade B or more, CRC, adenomas, ileal ulcers or gastro-duodenal peptic ulcers were considered to be relevant lesions while a mild gastritis or non-complicated hiatus hernia were not.

Disorders such as Child - Pugh C stage hepatic cirrhosis, advanced (stage IV or V) renal disease, thrombocytopenia or coagulation disorders were considered to be pro-hemorrhagic co- morbidities. Chronic non-steroidal anti-inflammatory drug (NSAID) use was defined as daily consumption during $\geq 1$ month within the 2 months before the fecal sampling.

\section{Statistical analysis}

Continuous variables with a normal distribution were reported as mean (standard deviation) and compared using the Student's $t$ test. Continuous variables with a skewed distribution were reported as median (interquartile range, IQR) and compared using the Mann-Whitney $U$ test. Frequencies (\%) were used to report categorical variables, which were compared using the Chi-squared test or Fischer's exact test when corresponding. All statistical tests were two-sided, and $P$ values $<0.05$ were considered to be statistically significant. Multiple logistic regression was used to identify independent predictors of PCCRC and relevant lesions using backward stepwise variable selection. All variables in the univariable analysis were included in the model. Odds ratios (ORs) and associated $95 \%$ confidence intervals $(\mathrm{Cls})$ were used to quantify the level of association. IBM SPSS Statistics for Windows, Version 23.0 (IBM Corp., Armonk, NY, United States) was used to analyze the data.

\section{Results}

A total of 130206 individuals (female $54.2 \%$; age $58.4 \pm 0.03$ years) were invited to the first screening round and 52731 $(40.5 \%)$ returned the FIT. Of them, 3065 (5.8\%) individuals had a positive result and 2659 (86.7\%) underwent colonoscopy. According to the result of this index colonoscopy, 179 (6.7\%) individuals presented CRC, 1637 (61.6\%) presented adenomas and 811 (30.5\%) (female 60.7\%; age $59.1 \pm 5.6$ ) had a negative colonoscopy. Out of 811 individuals with negative colonoscopy, $102(12.6 \%)$ had potentially bleeding lesions and the remaining 709 had a normal colonoscopy. $~$ Table 1 depicts the baseline

- Table 1 Baseline characteristics for the 811 individuals with negative colonoscopy.

\begin{tabular}{|l|c|c|c|}
\hline & $\begin{array}{l}\text { Normal colonoscopy } \\
\text { (n=709) }\end{array}$ & $\begin{array}{l}\text { Potentially bleeding lesions } \\
\text { (n=102) }\end{array}$ & P value \\
\hline Age (standard deviation), years & $58.9(5.6)$ & $60.4(5.2)$ & 0.012 \\
\hline Gender: female (\%) & $445(62.8)$ & $47(46.1)$ & 0.001 \\
\hline FIT, median (IQR), $\mu \mathrm{g} \mathrm{Hb} / \mathrm{g}$ feces & $47.0(79.8)$ & $72.9(208.7)$ & 0.004 \\
\hline Pro-hemorrhagic comorbidities (\%) & $7(1.0)$ & $1(1.0)$ & 0.995 \\
\hline - Advanced hepatic disease (\%) & $3(0.4)$ & 0 \\
\hline - End-stage renal disease (\%) & $3(0.4)$ & $1(1.0)$ \\
\hline - Coagulation, platelets or blood vessel disorders (\%) & $1(0.1)$ & $17(16.7)$ \\
\hline Concomitant therapy (\%) & $73(10.3)$ & $10(9.8)$ \\
\hline - Antiplatelets (\%) & $56(7.9)$ & $1(1.0)$ \\
\hline - Anticoagulants (\%) & $11(1.6)$ & $6(5.9)$ \\
\hline - NSAIDs (\%) & $6(0.8)$ & 0.236 \\
\hline FIT, fecal immunochemical test; IQR, interquartile range; NSAIDs, non-steroidal anti-inflammatory drugs. & 0.056 \\
\hline
\end{tabular}




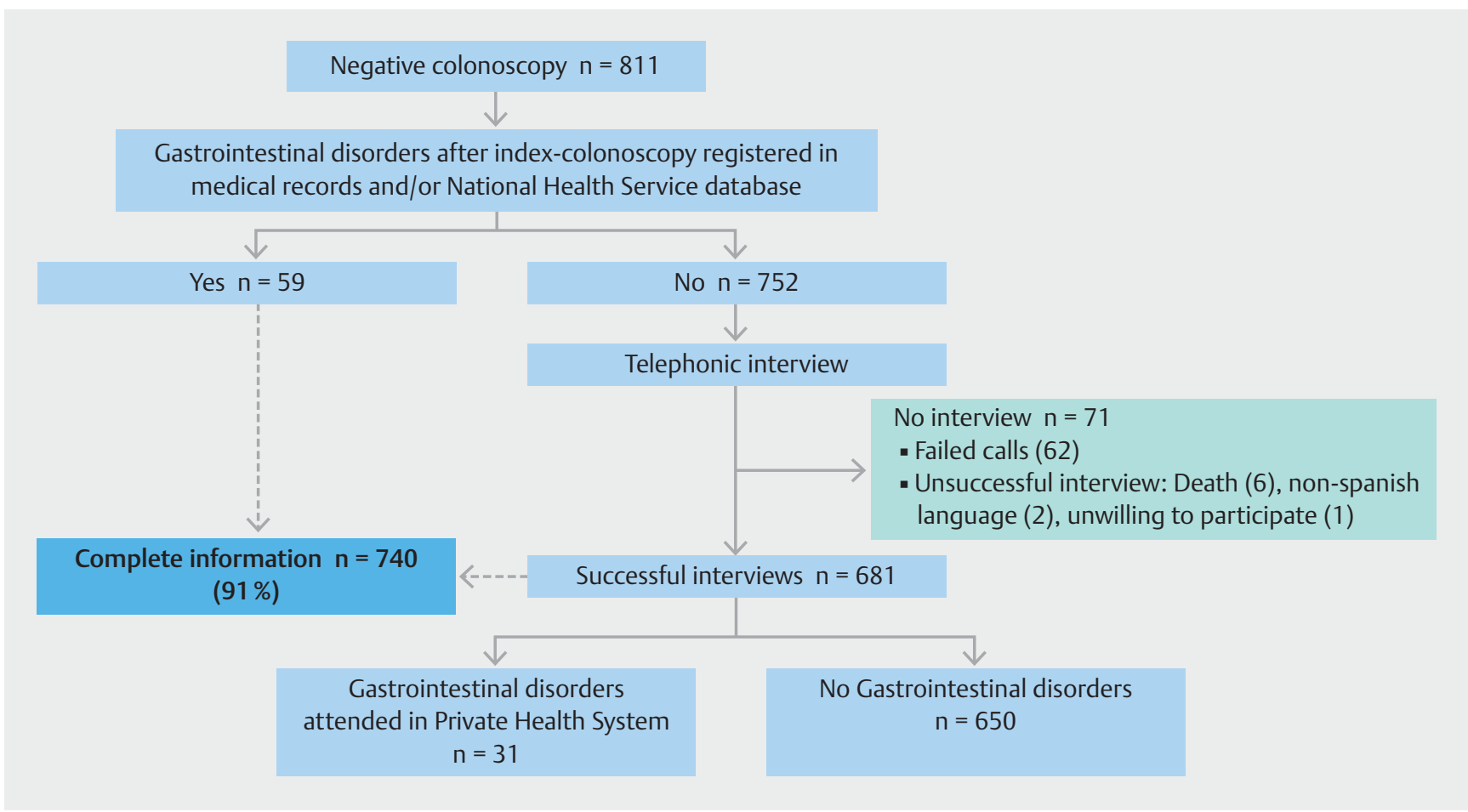

Fig. 1 Flow chart of data acquisition for the 811 individuals with negative colonoscopy.

characteristics of these individuals. Individuals with potentially bleeding lesions were slightly older with a higher proportion of men $(62.8 \%)$ and chronic NSAIDs consumers than those with normal colonoscopy. As expected, these individuals had higher hemoglobin concentrations in feces than those with normal colonoscopy ( $\vee$ Table 1$)$.

As is shown in $\mathbf{F i g .} 1$ a complete follow-up was available in 740 (91\%) of the individuals with negative colonoscopy with an average observation time of 4.7 years (range, $3.5-6.3$ years). - Fig. 2 summarizes the outcomes for the 811 individuals with negative colonoscopy.

\section{Incidence of PCCRC}

Three out of 740 individuals developed PCCRC (age, $56.3 \pm 7.5$ years; $66 \%$ men) at 11,27 , and 28 months after the negative colonoscopy ( $\mathbf{F i g . 2}$ ), resulting in a cumulative incidence of $0.4 \%$ and an incidence rate of $0.8 / 1000$ person-years. Furthermore, the rate of PCCRC among the total number of cancers detected in the cohort who underwent the index colonoscopy was $1.65 \%$ (3/182).

All tumors were TNM stage III, had normal expression of mismatch repair proteins at immunohistochemistry, and had no microsatellite instability. The patient who developed PCCRC at 11 months had a polypoid lesion of $15 \mathrm{~mm}$ in the ascending colon, whereas the other two individuals had larger tumors in the sigmoid colon and rectum. None of these three patients had synchronous neoplastic lesions. Regarding index colonoscopies, all were reported as complete with an adequate bowel preparation. Unfortunately, an exhaustive post-hoc revision of the index colonoscopy was not possible since screening colo- noscopies were not systematically video recorded. Details of the three individuals with PCCRC are shown in $>$ Table 2 . There were no statistically significant differences with regard to age, gender, FIT level, comorbidities, and chronic use of antiplatelets/anticoagulants or NSAIDs among individuals with or without PCCRC (data not shown).

\section{Other findings during follow-up}

As shown in $>$ Fig. 2, among those 647 individuals with normal index colonoscopy, 70 (11\%) presented gastrointestinal disorders that required complementary endoscopic procedures. In 32 patients, relevant lesions were detected, of which 11 were located in the upper gastrointestinal tract and 21 in the terminal ileum or colon - rectum (including the three cases of PCCRC). The remaining 38 individuals had a normal endoscopic examination.

One-third of relevant lesions (11 out of 32) were significant neoplasias: two $(2 / 647 ; 0.3 \%)$ located in the upper-medium gastrointestinal tract (gastric cancer and small-bowel lymphoma, respectively) and nine (9/647; $1.4 \%$ ) located in the colonrectum ( 6 advanced adenomas, 3 invasive CRC). The remaining relevant lesions (21 out of 32 ) were inflammatory, vascular disorders, or non-advanced colorectal adenomas. Four out of 32 relevant lesions were diagnosed within the first 6 months: an advanced gastric adenocarcinoma clinically manifested by hematemesis and melena; a caustic esophageal stricture, with monthly esophageal dilations due to recurrent dysphagia; an advanced jejunal lymphoma, manifested by weight loss and intestinal occlusion; and a gastric antral vascular ectasia manifested by iron-deficiency anemia ( $\triangleright$ Fig. 2). 
- Table 2 Characteristics of the three individuals with PCCRC.

\begin{tabular}{|c|c|c|c|}
\hline & Patient 1 & Patient 2 & Patient 3 \\
\hline \multicolumn{4}{|l|}{ General characteristics } \\
\hline - Gender & Female & Male & Male \\
\hline - Age, years & 65 & 53 & 51 \\
\hline - FIT, $\mu \mathrm{g} \mathrm{Hb/g} \mathrm{feces}$ & 68 & 44 & 90.6 \\
\hline \multicolumn{4}{|l|}{ Index colonoscopy } \\
\hline - Findings & Normal & $\begin{array}{l}\text { Distal diminutive hyperplastic polyps } \\
\text { and diverticulosis }\end{array}$ & Normal \\
\hline - Bowel preparation & Excellent & Good & Good \\
\hline \multicolumn{4}{|l|}{ Subsequent colonoscopy } \\
\hline - Elapsed time since index colonoscopy, months & 11 & 27 & 28 \\
\hline - Symptoms & Change in bowel habits & Rectal bleeding & Rectal bleeding \\
\hline \multicolumn{4}{|l|}{ Tumor characteristics } \\
\hline - Location & Ascending colon & Sigmoid colon & Rectum \\
\hline " Morphology & Sessile lesion $15 \mathrm{~mm}$ & Stenosing tumor & Flat lesion $40 \mathrm{~mm}$ \\
\hline - Stage at diagnosis (TNM) & $\mathrm{III} A=\mathrm{T} 1-2 \mathrm{~N} 1 \mathrm{M} 0$ & $\mathrm{IIIB}=\mathrm{T} 2-3 \mathrm{~N} 2 \mathrm{M} 0$ & $I I I A=T 1-2 \mathrm{~N} 1 \mathrm{M} 0$ \\
\hline " Histology & Low grade & $\begin{array}{l}\text { High grade ( } 40 \% \text { mucinous component, } \\
\text { signet ring cells) }\end{array}$ & Moderate grade \\
\hline - Expression of MMR protein & Normal & Normal & Normal \\
\hline Microsatellite instability & No & No & No \\
\hline
\end{tabular}

Factors such as age, gender, FIT level, and chronic use of antiplatelets or anticoagulants were not associated with a higher incidence of relevant lesions. However, in the multivariable analysis, those individuals with either pro-hemorrhagic advanced comorbidities or chronic use of NSAIDs presented a higher risk of having a relevant lesion (OR 16 [3.5-79.1]; $P<0.001$ and OR 11 [1.9-64.0]; $P=0.006$, respectively) ( $\triangleright$ Table 3 ). The subsequent relevant lesions in the two NSAID users were ileal aphthous ulcers and sigmoidal colitis, respectively. A gastric antral vascular ectasia was found in a patient with advanced hepatic cirrhosis. Finally, a jejunal lymphoma and one advanced adenoma (in situ carcinoma) were found in two patients with endstage renal disease ( $\triangleright$ Table 3$)$.

Potentially bleeding lesions at index colonoscopy were considered to be responsible for the FIT positivity in this group of individuals (> Fig. 2).

\section{Discussion}

This observational study reports the outcome of individuals with positive FIT and negative colonoscopy recruited in an organized, population-based CRC screening program after an average follow-up period of 4.7 years (range, $3.5-6.3$ years) and exhaustive data collection.

Three out of 740 individuals with negative colonoscopy developed PCCRC, resulting in a cumulative incidence of $0.4 \%$ and an incidence rate of $0.8 / 1000$ person-years. Furthermore, the rate of PCCRC among the total number of all detected CRC in the cohort (screen-detected plus PCCRC) was $1.65 \%$ (3/182).

The reported proportions of interval CRC vary greatly, ranging from $0.8 \%$ [19] of colonoscopic examinations to up to $9 \%$ [20] of all diagnosed CRCs; they are not comparable due to the use of different calculation methods [7,8]. The incidence proportion of PCCRC/number of colonoscopies could be a more practical method for assessing the quality of a colonoscopy unit but currently there is not a standardized method.

The incidence of CRC after a negative colonoscopy in the context of population organized FOBT-based screening programs has only been reported in two studies that used guaiacFOBT. A Danish study reported 14 CRCs out of 771 positive guaiac-FOBT followed by a negative colonoscopy (1.8\%) after 8 years of follow-up [11]. In that study, the individuals with positive FOBT and subsequent negative colonoscopy had the same long-term CRC risk as individuals with positive FOBT and adenomas and as the unscreened reference population [11]. Based on these results, the authors suggested that a 10-year interval of screening may not be safe. Another recent study from a Scottish population screening program reported a lower rate of PCCRC or missed cancers $(0.3 \%, 0.9 \%$, and $0.5 \%$ in the first, second, and third round, respectively, after 2 years of followup) [6]. Unfortunately, these proportions were not comparable to ours because of different follow-up times. 


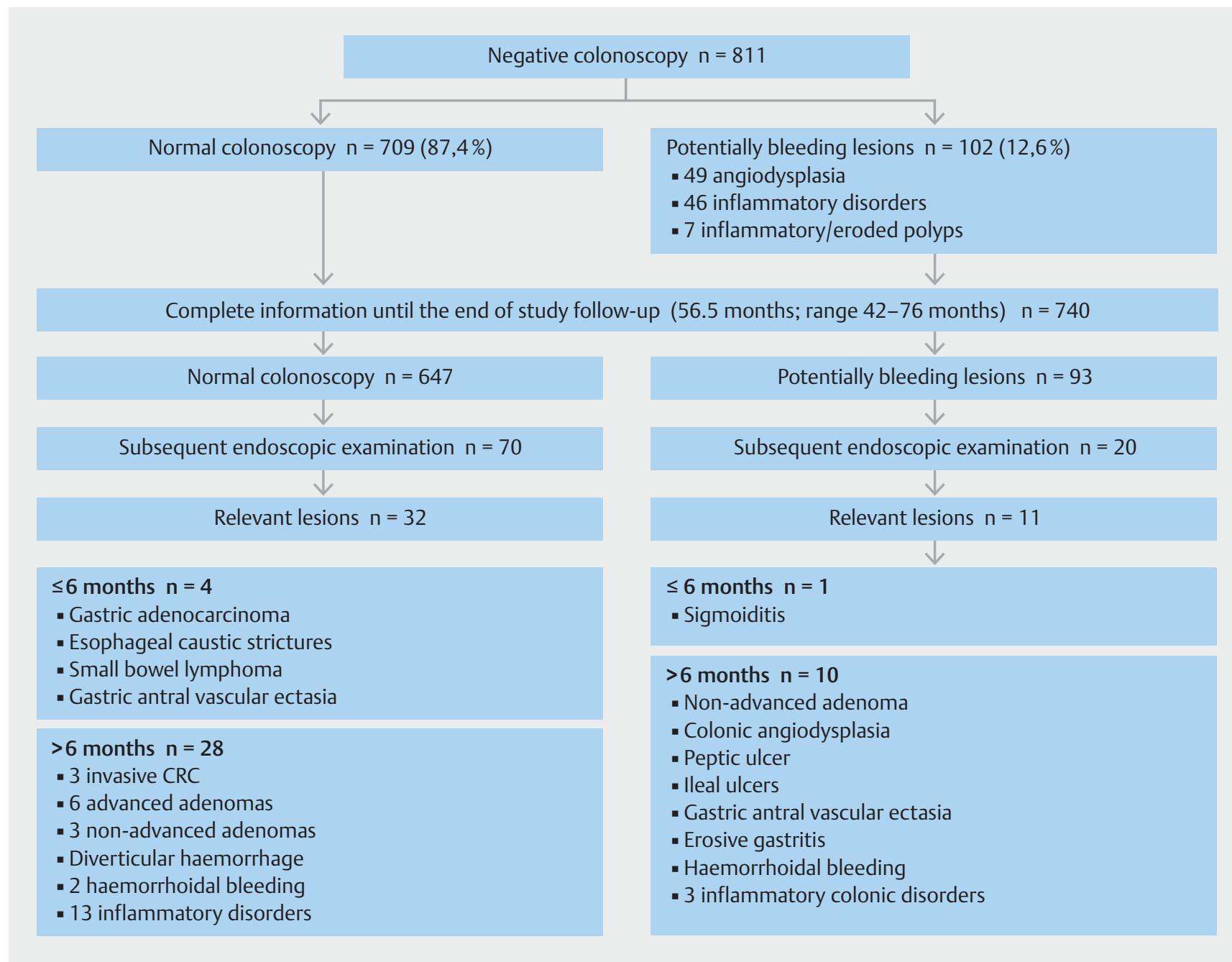

- Fig. 2 Outcomes for the 811 individuals with negative colonoscopy.

The low incidence and characteristics of PCCRC found in our FIT-based screening cohort reinforce the concept that quality of colonoscopy is of paramount importance $[5,21,22]$. In our cohort, all three patients presented with stage III CRC and had normal mismatch repair protein expression and microsatellite stability, thus excluding a potential accelerated carcinogenesis [23]. It is highly suggestive that they may correspond to missed lesions at the index colonoscopy. The first lesion was a $15 \mathrm{~mm}$ sessile polyp located in the ascending colon. Proximal location is a recognized risk factor for interval cancer because flat and serrated lesions are more likely to arise in this area and which are easier to miss $[24,25]$. The second one was a stenosing tumor in the sigmoid colon in a patient with diverticula. Previous reports have shown that the presence of diverticula and a spastic sigmoid colon might lead to an inadequate exploration of the fold of the colon and large polyps might be undiagnosed [24]. The third lesion arose in a laterally spreading tumor in the rectum, a location where blind spots are present without retroflexion. In such a context, the endoscopist's adenoma detection rate is the main quality indicator directly associated with the risk for interval cancers [22]. Unfortunately, we did not have individual (per-endoscopist) adenoma detection rates at that time, and withdrawal time and rectal retroflection were not reported and video-recordings of the colonoscopies were not available. Continuous efforts to improve quality in colonoscopy technique are required in order to strengthen the preventive role of current screening strategies.

Our results also highlight specific situations in individuals with a positive FIT and a negative colonoscopy, which deserve some comments. Firstly, with regard to anatomical location of the subsequent relevant lesion, one-third of them were located in the upper gastrointestinal tract. The FIT a priori does not detect digested blood because it uses antibodies directed against human intact globin epitopes. Thus, the positivity due to upper gastrointestinal bleeding lesions is controversial but it cannot be definitively ruled out. Physiologically, the hemoglobin is cleaved to form heme and globin by gastric pepsin protease, pancreatic proteases or both in the upper gastrointestinal tract [26]. Part of the hematin is absorbed and the remainder is degraded mainly by colonic bacteria to form porphyrins. The globin is digested by pepsin, pancreatic and intestinal proteases to yield peptides and amino acids [26]. However, alterations in the 
- Table 3 Univariable and multivariable analysis of factors related to subsequent relevant lesion in individuals with normal colonoscopy.

\begin{tabular}{|c|c|c|c|c|c|c|}
\hline & \multicolumn{6}{|l|}{ Normal colonoscopy } \\
\hline & $\begin{array}{l}\text { No relevant lesion } \\
(n=615)\end{array}$ & $\begin{array}{l}\text { Relevant lesion } \\
(n=32)\end{array}$ & $\begin{array}{l}\text { Univariable } \\
\text { analysis, OR }\end{array}$ & $P$ value & $\begin{array}{l}\text { Multivariable analy- } \\
\text { sis }^{1} \text {, adjusted OR }\end{array}$ & $P$ value \\
\hline $\begin{array}{l}\text { Age, mean } \pm \text { standard } \\
\text { deviation, years }\end{array}$ & $58.6 \pm 5.5$ & $59.3 \pm 5.8$ & - & 0.474 & - & - \\
\hline Gender, female (\%) & $386(56.3)$ & $18(56.3)$ & $1.31(0.64-2.68)$ & 0.458 & - & - \\
\hline $\begin{array}{l}\text { FIT, median (IQR), } \\
\mu \mathrm{g} \mathrm{Hb} / \mathrm{g} \text { feces }\end{array}$ & $46.4(29.7-112.2)$ & $47.9(24.4-90.3)$ & - & 0.673 & - & - \\
\hline NSAIDs (\%) & $4(0.7)$ & $2(6.3)$ & $10.81(1.7-57.81)$ & 0.031 & $11.2(1.9-64.0)$ & 0.006 \\
\hline Antiplatelets (\%) & $44(7.2)$ & $4(12.5)$ & $1.85(0.62-5.52)$ & 0.286 & - & - \\
\hline Anticoagulants (\%) & $11(1.8)$ & 0 & - & - & - & - \\
\hline $\begin{array}{l}\text { Advanced chronic } \\
\text { disease (\%) }\end{array}$ & $4(0.7)$ & $3(9.4)$ & $17.4(3.7-81.3)$ & 0.003 & $16.8(3.5-79.1)$ & $<0.001$ \\
\hline
\end{tabular}

digestive processes may facilitate the identification of globin by the FIT [26]. For example, the hemoglobin degradation may be retarded in cases of marked reduction in gastric acid secretion (i. e. vagotomy, gastrectomy or drugs) because a gastric $\mathrm{pH}$ below 4 is required to convert pepsinogen in the activated pepsin $[27,28]$. In a study of participants in a FIT-based CRC screening program [29], the use of proton pump inhibitors was identified as an independent factor for false positive results (OR, 1.8; $95 \%$ $\mathrm{Cl}, 1.1-2.9)$. Likewise, other factors such an accelerated stool transit time or the intrinsic pattern of bleeding of a certain gastrointestinal lesion (e.g. irregular flow and amount of blood) could also lead to a flow of small quantities of intact blood into the colon [26]. Thus, since FIT can detect quantities as low as $0.3 \mathrm{~mL}$ of blood in stool [30], positivity due to lesions above the colon might be reasonably accepted. In our cohort, these mechanisms might explain a limited number of cases, mainly those with active bleeding lesions detected within the first 6 months after the index colonoscopy. However, according to our results and in consonance with previous studies [31-33], the likelihood of having a relevant asymptomatic lesion in the upper gastrointestinal tract is too low to support systematically performing an upper endoscopy in these individuals.

Secondly, in our study, advanced comorbidities and chronic use of NSAIDs were significantly associated with a higher likelihood of having a subsequent relevant lesion. The use of NSAIDs has previously been described as a risk factor for a false positive FIT [34]. These factors may cause transient inflammatory lesions or increase the physiologic gastrointestinal bleeding and cause the false positive result.

Finally, the vast majority ( $95 \%$ ) of individuals did not present subsequent gastrointestinal events after a 3- to 5-year followup period. The reasons for positivity remain elusive but several hypotheses can be proposed. Despite explicit instructions about stool sample collection, it is difficult to ensure an adequate compliance in cases of macroscopic bleeding. The elapsed time since a positive FIT result until colonoscopy was 1 to 2 months. Therefore, it is possible that bleeding inflammatory lesions might have disappeared at the time of examination. Also it is worthwhile mentioning that there is a physiologic gastrointestinal blood loss through the small bowel of 0.5 to $1.5 \mathrm{~mL}$ per day [26] that might be detected by the FIT if physiologic mechanisms are altered.

We are aware of some limitations of the study. Firstly, inaccessibility to a centralized nationwide health registry for data cross-referencing may lead to missing information. However, the characteristics of index colonoscopy are derived from the screening program database, in which data are prospectively collected. In addition, follow-up information was exhaustively completed through medical records, regional health service databases, and successful telephonic interviews in $91 \%$ of all patients ( $\bullet$ Fig. 1). Secondly, data collection after the index colonoscopy was based on medical consultation and endoscopic procedures, which were performed according to predominant symptoms rather than systematically assessed using a pre-established research protocol. We also acknowledge that a subgroup of patients might have not had "enough" follow-up, considering that the mean sojourn time of preclinical cancer progressing to a detected cancer ranged from 4.5 to 5.8 years [35]. Thirdly, non-endoscopic or more primary care lesions such as hemorrhoids and fissures might be missed. Moreover, it may be questionable how carefully these minor lesions are documented in the colonoscopy report. Fourthly, since a comparison with individuals with negative FIT is lacking, in some cases, it is difficult and perhaps daring to consider a subsequent relevant lesion as a cause of previous FIT positivity.

In conclusion, in an organized population FIT-based CRC screening program, the cumulative incidence of CRC after a negative colonoscopy was very low $(0.4 \%)$, but not zero. Accordingly, continuous efforts are required to improve quality standards of colonoscopy in order to strengthen the preventive 
role of the current CRC screening strategies. On the other hand, the vast majority ( $95 \%$ ) of these individuals did not present any subsequent relevant lesion related to FIT positivity. Thus, our results do not support systematically performing additional endoscopic procedures in those individuals. From a practical point of view, although a positive FIT preselects individuals who benefit from more invasive testing because of their high risk of presenting colorectal neoplastic lesions, once a high quality colonoscopy has been performed, the likelihood of missing relevant lesions is negligible.

\section{Acknowledgments}

This work was supported by grants from the Instituto de Salud Carlos III (PI12/01481), Fundación Científica Asociación Española Contra el Cáncer (GCB13131592CAST), Ministerio de Economía y Competitividad (SAF2014-54453-R), Agència de Gestió d'Ajuts Universitaris i de Recerca (2014SGR135), and from the crowdfunding "Amics del Clínic". This work was cofunded by the European Regional Development Fund (ERDF). CIBEREHD is funded by the Instituto de Salud Carlos III.

\section{Competing interests}

\section{None}

\section{References}

[1] Kahi C], Imperiale TF, Juliar BE et al. Effect of screening colonoscopy on colorectal cancer incidence and mortality. Clin Gastroenterol Hepatol 2009; 7: $770-775$

[2] Hardcastle JD, Chamberlain JO, Robinson MH et al. Randomised controlled trial of faecal-occult-blood screening for colorectal cancer. Lancet 1996; 348: 1472 - 1477

[3] Zorzi M, Fedeli U, Schievano E et al. Impact on colorectal cancer mortality of screening programmes based on the faecal immunochemical test. Gut 2015; 64: $784-790$

[4] Imperiale TF, Glowinski EA, Lin-Cooper C et al. Five-year risk of colorectal neoplasia after negative screening colonoscopy. NEJM 2008; 359: $1218-1224$

[5] Brenner H, Chang-Claude J, Seiler CM et al. Long-term risk of colorectal cancer after negative colonoscopy. J Clin Oncol 2011; 29: 3761 3767

[6] Steele RJC, McClements P, Watling C et al. Interval cancers in a FOBTbased colorectal cancer population screening programme: implications for stage, gender and tumour site. Gut 2012; 61: 576-581

[7] Morris EJA, Rutter MD, Finan PJ et al. Post-colonoscopy colorectal cancer (PCCRC) rates vary considerably depending on the method used to calculate them: a retrospective observational populationbased study of PCCRC in the English National Health Service. Gut 2015; 64: $1248-1256$

[8] Sanduleanu S, le Clercq CMC, Dekker E et al. Definition and taxonomy of interval colorectal cancers: a proposal for standardising nomenclature. Gut 2015; 64: 1257 - 1267

[9] Carroll MRR, Seaman HE, Halloran SP. Tests and investigations for colorectal cancer screening. Clin Biochem 2014; 47: 921 - 939
[10] Raginel T, Puvinel J, Ferrand O et al. A population-based comparison of immunochemical fecal occult blood tests for colorectal cancer screening. Gastroenterology 2013; 144: 918 - 925

[11] Bjerrum A, Andersen O, Fischer A et al. Long-term risk of colorectal cancer after negative colonoscopy in a Danish gFOBT screening cohort. Int J Cancer 2017; 141: 503-511

[12] Lieberman DA, Rex DK, Winawer S] et al. Guidelines for colonoscopy surveillance after screening and polypectomy: a consensus update by the US Multi-Society Task Force on Colorectal Cancer. Gastroenterology 2012; 143: $844-857$

[13] van Rijn JC, Reitsma JB, Stoker J et al. Polyp miss rate determined by tandem colonoscopy: a systematic review. Am J Gastroenterol 2006; 101: $343-350$

[14] Kaminski MF, Wieszczy P, Rupinski M et al. Increased rate of adenoma detection associates with reduced risk of colorectal cancer and death. Gastroenterology 2017; 153: 98-105

[15] Rivero-Sánchez L, Pellisé M. [Bowel preparation for colonoscopy. Any significant progress on the horizon?] Gastroenterol Hepatol 2015; 38 : $287-300$

[16] Jover R, Herráiz M, Alarcón O. et al. Spanish Society of Gastroenterology (AEG) and Spanish Society of Gastrointestinal Endoscopy (SEED) Working Group. Clinical practice guidelines: quality of colonoscopy in colorectal cancer screening. Endoscopy 2012; 44: 444 - 451

[17] Quintero E, Castells A, Bujanda L et al. Colonoscopy versus fecal immunochemical testing in colorectal-cancer screening. NEJM 2012; 366: $697-706$

[18] Jover R, Zapater P, Polanía E et al. Modifiable endoscopic factors that influence the adenoma detection rate in colorectal cancer screening colonoscopies. Gastrointest Endosc 2013; 77: 381 -389.e1

[19] Rex DK, Bond JH, Feld AD. Medical-legal risks of incident cancers after clearing colonoscopy. Am J Gastroenterol 2001; 96: 952 - 957

[20] Baxter NN, Sutradhar R, Forbes SS et al. Analysis of administrative data finds endoscopist quality measures associated with postcolonoscopy colorectal cancer. Gastroenterology 2011; 140: 65 - 72

[21] Rembacken B, Hassan C, Riemann JF et al. Quality in screening colonoscopy: position statement of the European Society of Gastrointestinal Endoscopy (ESGE). Endoscopy 2012; 44: 957 - 968

[22] Kaminski MF, Regula J, Kraszewska E et al. Quality indicators for colonoscopy and the risk of interval cancer. NEJM 2010; 362: 1795-1803

[23] Zaanan A, Meunier K, Sangar F et al. Microsatellite instability in colorectal cancer: from molecular oncogenic mechanisms to clinical implications. Cell Oncol (Dordr) 2011; 34: 155-176

[24] Singh S, Singh PP, Murad MH et al. Prevalence, risk factors, and outcomes of interval colorectal cancers: A systematic review and metaanalysis. Am J Gastroenterol 2014; 109: 1375-1389

[25] Kahi C], Hewett DG, Norton DL et al. Prevalence and variable detection of proximal colon serrated polyps during screening colonoscopy. Clin Gastroenterol Hepatol 2011; 9: 42 - 46

[26] Rockey DC. Occult gastrointestinal bleeding. NEJM 1999; 341: 38 - 46

[27] Samloff IM. Peptic ulcer: the many proteinases of aggression. Gastroenterology 1989; 96: 586-595

[28] Wang J, Barbuskaite D, Tozzi M et al. Proton pump inhibitors inhibit pancreatic secretion: Role of gastric and non-gastric $\mathrm{H}+/ \mathrm{K}+$-ATPases. PLoS One 2015; 10: e0126432

[29] Ibáñez-Sanz G, Garcia M, Rodríguez-Moranta F et al. Prescription drugs associated with false-positive results when using faecal immunochemical tests for colorectal cancer screening. Dig Liver Dis 2016; 48: $1249-1254$

[30] Saito H. Screening for colorectal cancer by immunochemical fecal occult blood testing. Jpn J Cancer Res 1996; 87: 1011-1024 
[31] Ng JY, Chan DKH, Tan KK. Is gastroscopy for fecal immunochemical test positive patients worthwhile? Int J Colorectal Dis 2017; 32: 95 98

[32] Chiang T-H, Lee Y-C, Tu C-H et al. Performance of the immunochemical fecal occult blood test in predicting lesions in the lower gastrointestinal tract. Can Med Assoc J 2011; 183: 1474-1481

[33] Choi JS, Choi JY, Cho HG et al. Is esophagogastroduodenoscopy necessary in patients with positive fecal occult blood tests and negative colonoscopy? Scand J Gastroenterol 2013; 48: 657-662
[34] Stegeman I, de Wijkerslooth TR, Stoop EM et al. Risk factors for false positive and for false negative test results in screening with fecal occult blood testing. Int J Cancer 2013; 133: 2408-2414

[35] Brenner H, Altenhofen L, Katalinic A et al. Sojourn time of preclinical colorectal cancer by sex and age: Estimates from the German national screening colonoscopy database. Am J Epidemiol 2011; 174: 1140 1146 